\title{
Internal Strategies for Assessing Organizational Communication Channel Effectiveness
}

\author{
Melvin E. Murphy \\ Northern Virginia Community College \\ Douglas G. Campbell \\ Walden University \\ Denise L. Land \\ Walden University
}

Evolving communication technology, the increased volume of information needed by businesses and the intensified competitive environment have made internal company communication more critical to business success. The purpose of this multiple case study was to explore strategies for assessing the effectiveness of internal communication channels used by managers and leaders of three law firms in the Commonwealth of Virginia. The participating firms were recognized by their peers as being exceptionally well managed. Data from interviews and company documents were analyzed through the conceptual lens of channel expansion theory and the use of software coding to identify patterns and themes. Three important themes emerged: informal assessment strategies, indirect assessment strategies, and efficient versus timely assessments. The first theme suggested the effectiveness of an informal assessment strategy, depending on the size and complexity of the organization. The second theme reflected the effectiveness of an indirect assessment for organizations that lack the resources to support a more direct and specific assessment process. The third theme reflected the participants' perceptions of informal and indirect assessments may be more efficient, the feedback from the assessments are often less timely. Managers may consider these themes in formulating communication policies. Small professional service firms, such as legal firms, provide important services to individuals, families, and businesses in their community.

Keywords: internal communications, communication channels, organizational communication channels, strategic communication, communication channel effectiveness

\section{Introduction}

For as long as any of us can remember, organizational communications have primarily been assessed based on the content of the message. The standard thinking was, and still is in most organizations, that a well-crafted message is successful regardless of the means (channels) of delivery. Some consideration was given to a speaker's skill in delivering a spoken message, and consideration was given to the intended audience, but the content of the message remained the primary focus when assessing the potential effectiveness of a message. There are several implications of the findings of this study that make it unique among published research. This study appears to be the first to indicate that the assessment of the effectiveness and efficiency of the means of internal organizational communication (channels) is as important as the assessment of the content of the 
message. Additionally, we found that the content of a message and the anticipated communication channels are interdependent, in that the anticipated message channels affect the message content and vice versa. Additionally, expanding an organization's technology-based internal communication channels can be a competitive advantage and a cost effective investment, if they are properly maintained and assessed.

\section{Purpose, Population, and Data Collection}

The purpose of this qualitative multiple case study was to explore what internal strategies, if any, that leaders of medium-sized law firm used to assess the effectiveness of their organizations' internal communication channels. Also captured were key employees' perceptions of the effectiveness of specific intraorganizational communication channels, and of the existing strategies used for assessing the effectiveness of those channels.

The selected populations for this study were three medium-size law firms located in the Commonwealth of Virginia, each having approximately 50 employees and a communications department. Radley and Chamberlain (2012) and Rubin and Rubin (2012) found that purposeful sampling for interviews of employees enabled researchers to elicit richer data based on the descriptive experiences that accumulate from long-term employment. A purposeful sampling approach, therefore, corresponded to the data collection design for this study. Additionally, we reviewed corporate communications documents. However, each organization lacked formal communication plans to support the operations of their internal communication departments; thus, we primarily relied on data from interviews. The three organizations that participated in this research were considered exceptionally well managed and well organized by peers in their field.

The criterion for participation was executives or marketing managers, communication managers, and office managers recruited from each selected case firm based on the expectation that this participant pool would allow sufficient data collection for data saturation. Potential participants were selected by each case firm's office manager based on the manager's knowledge of employees who had daily responsibilities to disseminate company communications. We then selected those who would be actual participants. In accordance with the recommendations of Yin (2009), Nunan and Yenicioglu (2013), and Plankey-Videla (2012), participant interviews were conducted in neutral locations (i.e., coffee shops, public libraries, restaurants) to allow participants to feel more comfortable about responding to research questions. Results emerged in the form of themes that the leaders of similarly sized professional organizations can use when developing their organizations' internal communications strategies.

\section{Literature Review}

Most published literature focused on the conduct, use, or management of only one specific communication channel. Less common was published research that focused on systems of internal organizational communication channels. Even less common was published research that focused on the assessment of organizational communication channels. The relevant literature revealed the existence of two opposing positions. Corporate leaders tended to believe that assessing the effectiveness of the organization's communication channels was either unnecessary or a low priority (Jimenez-Castillo \& Sanchez-Perez, 2013; Men, 2014; Tenhiälä, \& Salvador, 2014). Opposing this opinion were the communication researchers who supported greater managerial interest in the relative effectiveness of organizational communication channels (Bronn, 2013; Castle \& Bourne, 2016; Neves \& Eisenberger, 2012). We took these contradictory opinions as evidence of the potential 
value of this study. Specifically, the existence of two opposing positions validated the need for a disciplined exploration of functional strategies to assess the effectiveness of organizational communication channels.

\section{Conceptual Framework}

Channel expansion theory (CET) formulated by Carlson \& Zmud (1999), was the conceptual framework for this study. The publishing of this theory opened the door among researchers to the discussion of the significance of the profusion of new communication channels to message content. CET represented a potential explanation and prediction of how employees' perceptions about communication channel effectiveness, as well as how users chose and assessed the effectiveness of communications channels. According to Carlson and Zmud, the four fundamental constructs underlying CET are employees' perceptions of and experience with (a) media use experience with a company's communication channels, (b) understanding of disseminated business messages, (c) experience with the internal culture of the company, and (d) working relationships with other managers, staff, and users of communication channels. As the conceptual framework of this current study, CET informed the development of the interview questions and served as the lens through which data was analyzed. Figure 1 illustrates the CET conceptual framework.

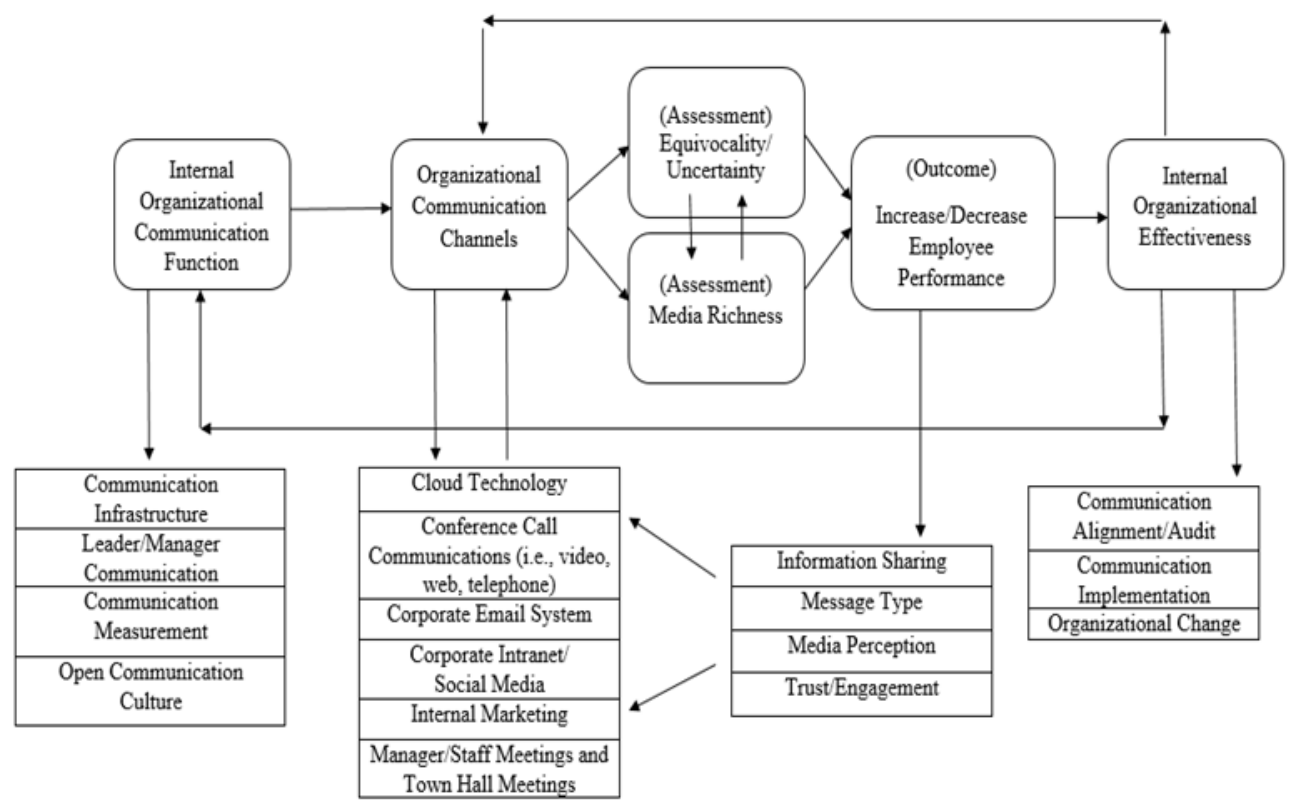

Figure 1. Graphical Representation of Channel Expansion Theory

The central aspect of CET in the context of this study was that a users' perceptions of communication channel effectiveness leads the users to change how the channel is used over time, which, under certain conditions, leads to the users expanding the use of the communications channel to achieve more effective communications (Carlson \& Zmud, 1999). 


\section{Data Analysis}

Both transcript review and member checking were used to increase the reliability and validity of data captured during participant interviews. The data was then organized, coded, and entered into a qualitative data analysis program. Pattern matching served as the basis for organizing and summarizing common themes in participants' responses (Yin, 2009). More specifically, we used cross-case analysis to (a) compare case-specific factors, (b) determine patterns of associations, and (c) generate coding tables and matrices. The data analysis process included compilation, coding, and data triangulation.

\section{Findings}

The overarching research question was: What internal strategies did managers of medium-sized law firms use, if any, to assess the effectiveness of their internal communication channels? After the successful completion of the data collection process, transcript review, and member checking, three core themes emerged from the data analysis: (a) informal assessment strategies, (b) indirect assessment strategies, and (c) timely versus efficient assessments.

\section{Theme 1: Informal Assessment Strategies}

An informal and unwritten communication channel assessment strategy can be effective and sufficient for some organizations. After reviewing the organizations' documents, policies, records, and the interview data, it was clear that the leaders of the three case firms had only informal and unwritten strategies to assess the effectiveness of the organizational communication channels. None of the organizations had any written formal communication plan or separate formal plan to assess the effectiveness of internal communication channels. While informal assessments appear functional, some participants thought a more formal and structured strategy may be beneficial as an organization grows or changes.

This finding contradicts the observations of Jimenez-Castillo and Sanchez-Perez (2013), who emphasized the importance of formal organizational communication channel assessments. This theme also contradicts Mishra, Boynton, and Mishra (2014), who found that assessing the efficiency of internal communication channels was important to ensuring effective internal communications. Jimenez-Castillo and Sanchez-Perez (2013) and Mishra et al. (2014) also emphasized that communication assessments help leaders to ensure that information is disseminated appropriately in their organization. Carlson and Zmud (1999) noted that some corporate executives and managers failed to provide internal strategies for assessing channel effectiveness because they lacked the needed knowledge and experience.

Table 1 shows the frequency of participants' comments about informal channel assessments. Case Firm A and Case Firm B participants discussed informal channel assessments 10 times, which was $10.6 \%$ of participant responses (see Table 1 ). 
Table 1. Number of Times Informal Assessment Discussed

\begin{tabular}{lcc}
\hline Theme Where Participant & & \\
Discussed Informal Assessment & Times Discussed & \% of Coverage \\
\hline LFA1 & 1 & $44 \%$ \\
LFA2 & 5 & $8 \%$ \\
LFA3 & 1 & $9 \%$ \\
LFA5 & 3 & $17 \%$ \\
LFB1 & 1 & $6 \%$ \\
LFB2 & 1 & $15 \%$ \\
LFB3 & 8 & $7 \%$ \\
Law firms' equivalent percentage numbers & 20 & $10.6 \%$ \\
\hline
\end{tabular}

Note. LFA = Law Firm A; LFB = Law Firm B. The number represents the participant.

\section{Theme 2: Indirect Assessments Strategies}

Indirect assessments of the effectiveness of internal communication strategies can be sufficient in some organizations. This theme contradicts the observations of Ryynänen, Jalkala, and Salminen (2013), who wrote that poorly constructed messages could be a barrier to an accurate assessment of the existing internal communication channels. This theme also contradicts the findings of Aggarwala and Subbian (2014), who found that directly assessing all aspects of organizational communication systems, including infrastructure and communication channels, resulted in benefits to both employees and the company. However, this finding is supported by the findings of Cobanica and Grecu (2014), who noted that assessing internal communications was a complex process, particularly the formulation of assessment policy for channel performance and for developing communication strategies. Table 2 shows the frequency of participants' comments about the continuous and varied indirect assessments. Both Case Firm A and Case Firm B participants discussed indirect assessment 11 times. Case Firm C discussed Indirect Assessment 1 time, which was 22.9\% of participant responses (see Table 2).

Table 2. Number of Times Indirect Assessment Discussed

\begin{tabular}{lcc}
\hline Theme Where Participant & & \\
Discussed Indirect Assessment & Times Discussed & \% of Coverage \\
\hline LFA1 & 2 & $19 \%$ \\
LFA2 & 3 & $11 \%$ \\
LFA3 & 2 & $16 \%$ \\
LFA4 & 2 & $30 \%$ \\
LFA5 & 2 & $14 \%$ \\
LFB1 & 3 & $34 \%$ \\
LFB2 & 2 & $21 \%$ \\
LFB3 & 6 & $41 \%$ \\
LFC1 & 1 & $43 \%$ \\
Law firms' equivalent percentage numbers & 23 & $22.9 \%$ \\
\hline
\end{tabular}

Note. LFA = Law Firm A; LFB = Law Firm B. The number represents the participant. 


\section{Theme 3: Efficient Versus Timely Assessments}

Some participants, while acknowledging the functionality of the informal and indirect approaches to channel assessment in place within the organization, expressed some frustration with those arrangements. Specifically, they desired more timely feedback and more timely corrective action than their present systems provided. Efficiency is the chief virtue of the informal and indirect strategies to the assessment of an organization's communications channels. Less time, effort, and costs (i.e. efficiency) appear to be the characteristics of the informal and indirect approaches. However, according to some participants, the tradeoff is a less than an optimal and timely system.

Specifically, some participants explained that the analysis by the management of the data appears to be slower than would be optimal, and slower than feedback from the informal and indirect assessment to the management team. Some of the participants seemed to assume that a more formal assessment regime would provide more timely feedback, although they were unable to provide a clear explanation of why they believed that to be so. The importance of timely assessments and the timely delivery of feedback to the management team was noted by Carlson and Zmud (1999) and Sprain and Boromisza-Habashi (2013).

The importance of timely assessments and the timely delivery of feedback to the management team was noted by Carlson and Zmud (1999) and Sprain and Boromisza-Habashi (2013). Table 3 shows the frequency of participants' comments about focusing on efficient and timely communications. Case Firm A participants focused on efficient and timely assessments seven times. Case Firm B participants focused on efficient and timely assessments five times. Case Firm C participants focused on efficient and timely assessments twice, which was $22.3 \%$ of participant responses (see Table 3).

Table 3. Number of Times Effective Versus Timely Communication Assessment Discussed

Theme Where Participant Discussed

Efficient Versus Timely Assessment Times Discussed \% of Coverage

\begin{tabular}{lcc} 
LFA1 & 1 & $10 \%$ \\
LFA2 & 1 & $4 \%$ \\
LFA3 & 1 & $9 \%$ \\
LFA4 & 2 & $33 \%$ \\
LFA5 & 2 & $16 \%$ \\
LFB1 & 3 & $37 \%$ \\
LFB2 & 1 & $11 \%$ \\
LFB3 & 1 & $8 \%$ \\
LFC1 & 2 & $95 \%$ \\
Law firms' equivalent percentage numbers & 14 & $22.3 \%$ \\
\hline
\end{tabular}

Note. LFA = Law Firm A; LFB = Law Firm B. The number represents the participant.

The findings revealed that midsized law firms, to varying degrees, may have informal channel assessment strategies. Formal channel assessments could be important for organizations. Additionally, the results indicated that having informal assessments or indirect assessments could be functional for organizations without the resources to establish a more rigid and costly formal assessment protocol. 


\section{Applications to Professional Practice}

The results of this study could assist leaders and managers of some small professional organizations with general communication planning, specifically regarding planning to assess the effectiveness of internal communication channels. Each theme provides insight into how similar organizations approached communication planning and channel assessment. The findings capture the participants' perspectives on both positive and negative aspects of their current communication processes. Managers of similar sized professional organizations who read this research will be better informed on the broader considerations of communication planning and specifically the assessment of internal communication channels. Ultimately, the reader may, because of this research, be better equipped to craft an optimal plan for the specific requirements and conditions of their organization.

\section{Recommendations for Action}

When developing an organization's communication plans, a mechanism for the assessment of the organization's communication channels should be a consideration. From Themes 1 and 2, it is evident that there are two foundational choices for leaders (a) the choice of a formal or informal approach to assessment of the organization's communication channels and (b) the choice of a direct or indirect approach to assessment. While most researchers who study communication planning and channel assessment clearly favor a formal and direct approach to assessment, Theme 3 revealed that there are benefits and consequences of each choice.

Almost any organizational leader is familiar with the concept of return on investment (ROI). Investment, in the context of ROI, refers to money directly spent but also to time and effort spent, which are also expenses. Return, in the context of ROI, refers to income and to organizational benefits that are often difficult to financially value. Much of managerial decision involves the consideration of ROI. We recommend that organizational leaders consciously evaluate the time and effort required for some form of formal and direct assessment of the internal organizational communications and consider the possible ROI of each option.

Specifically, organizational leaders should give consideration to the efficiency and effectiveness of their internal communication channels. The leaders should also consider the positive organizational benefits of being able to more effectively and rapidly institute improvements. If the costs, in time and money, exceed the ability of the current resources of the organization, then the leaders should consider developing the most effective and efficient assessment system that they can afford.

From the totality of this research comes the lesson that in the modern era of rapidly multiplying communication channels, leaders should give greater consideration to investing in communication planning and assessment. During the interviews, various participants bemoaned what they perceived as inadequate consideration and exploration in organizational communications infrastructure and planning. These participants, while acknowledging the functionality of their organization's current message delivery and assessment system also bemoaned that absence of current communication channels (i.e. social media) and more timely assessments.

Therefore, we recommend that leaders consider the broad benefits to employee morale of optimal communication alignment and assessment systems. Both the literature and the responses of some participants indicated that to some degree that there may be generational differences in perspectives about internal organizational communications. Those differences may be perceptions concerning the number and type of valid communication channels, and individuals' preferences for specific channels for specific tasks. These appear to be potential issues of employee morale, as well as missed 
organizational opportunities for improvement. The findings of this study and the recommendations can be used in professional services companies, industry conferences, corporate seminars, and workshops.

\section{Limitations}

It should be remembered that these research findings are based on qualitative data, from only three organizations, so the evidence cannot be considered conclusive to the degree that a confirmational quantitative study might be. Additionally, the responses and candidness of human participants may vary due to their position, concerns and perception of the interviewer. We made every effort to bring forth full commitment from the participants, and to present the finding and our conclusions as merely insightful and useful to practicing business professionals.

\section{Recommendations for Further Research}

We recommend further qualitative and quantitative research on postimplementation of various strategies for assessing communication channels. Such research could provide extant knowledge on the effectiveness and efficiency of those various strategies. We also recommend both qualitative and quantitative research to explore and examine how different groups within intergenerational workforce perceive the usefulness of various potential internal communication channels.

\section{Conclusion}

Law firm leaders and communication professionals who are interested in improving the effectiveness of their company's internal communications channels might find this study helpful in development of their organization's internal communication strategy and policies. Evolving communication technology, the increased volume of information needed by businesses, and the intensified competitive environment has made internal company communication more critical to a business's financial success. If this new knowledge is appreciated and put into practice by managers and leaders then there is the potential for radical change in how message content is designed, and increased interest in the use and maintenance of new technological communication channels. Thus, the findings can potentially affect positive change by proving how assessment of communication channels are administered and providing synergy for a more positive employee workplace experience, thus improving the quality of life for employees. The assessment strategies identified in this research might help guide leaders in operating more efficient and effective small professional service organizations that are better able to serve the needs of the local communities. Small professional service firms, such as legal firms, provide important services to individuals, families, and businesses in their community. The services they provide are critical to ensuring the safety and protection of their clients. 


\section{References}

Aggarwala, C., \& Subbian, K. (2014). Evolutionary network analysis: A survey. Association for Computing Machinery Computing Surveys, 47, 10. doi:10.1145/2601412

Carlson, J. R., \& Zmud, R. W. (1999). Channel expansion theory and the experiential nature of media richness perceptions. Academy of Management Journal, 42, 153-170. doi:10.2307/257090

Castle, J. D., \& Bourne, B. B. (2016). What leaders must know about communication channel selection. Journal of International Studies, 16, 13-26. doi:10.18374/JIMS-16-2.2

Cobanica, M., \& Grecu, I. (2014). System analysis of strategic communication at company's level. Economics, Management, and Financial Markets, 9, 230-238.

Bronn, P. S. (2013). How others see us: Leaders' perceptions of communication and communication managers. Journal of Communication Management, 18, 58-79. doi:10.1108/JCOM-03-20130028

Jimenez-Castillo, D., \& Sanchez-Perez, M. (2013). Integrated market-related internal communication: Development of the construct. International Journal of Market Research, 55, 563-585. doi:10.2501/IJMR-2013-048

Men, L. (2014). Strategic internal communication: Transformational leadership, communication channels, and employee satisfaction. Management Communication Quarterly, 28, 264-284. doi:10.1177/0893318914524536

Mishra, K., Boynton, L., \& Mishra, A. (2014). Driving employee engagement: The expanded role of internal communication. International Journal of Business Communication, 51, 183-202. doi:10.1177/232948814525399

Neves, P., \& Eisenberger, R. (2012). Management communication and employee performance: The contribution of perceived organizational support. Human Performance, 25, 452-464. doi:10.1080/08959285.2012.721834

Nunan, D., \& Yenicioglu, B. (2013). Informed, uninformed and participative consent in social media research. International Journal of Market Research, 55, 791-808. doi:10.2501/IJMR-2013-067

Plankey-Videla, N. (2012). Informed consent as a process: Problematizing informed consent in organizational ethnographies. Qualitative Sociology, 35, 1-21. doi:10.1007/s11133-011-9212-2

Radley, A. M., \& Chamberlain, K. (2012). The study of the case: Conceptualizing case study research. Journal of Community \& Applied Social Psychology, 22, 390-399. doi:10.1002/casp.1106

Rubin, H. J., \& Rubin, I. S. (2012). Qualitative interviewing: The art of hearing data (3rd ed.). Thousand Oaks, CA: Sage.

Ryynänen, H., Jalkala, A., \& Salminen, R. T. (2013). Supplier's internal communication network during the project sales process. Project Management Journal, 44, 5-20. doi:10.1002/pmj.21341

Sprain, L., \& Boromisza-Habashi, D. (2013). The ethnographer of communication at the table: Building cultural competence, designing strategic action. Journal of Applied Communication Research, 41, 181-187. doi:10.1080/00909882.2013.782418 
Tenhiälä, A., \& Salvador, F. (2014). Looking inside glitch mitigation capability: The effect of intraorganizational communication channels. Decisions Sciences, 45, 437-466. doi:10.1111/deci.12076

Yin, R. K. (2009). Case study research: Designs and methods (4th ed.). Thousand Oaks, CA: Sage.

The International Journal of Applied Management and Technology (IJAMT), sponsored by Walden University's School of Management, is a peer-reviewed, online journal that addresses contemporary national and international issues related to management and technology. The objectives of the IJAMT are to: (a) encourage collaborative and multi-disciplinary examinations of important issues in business and technology management, and (B) engage scholars and scholar-practitioners in a dynamic and important dialogue.

Walden University Publishing: http://www.publishing.waldenu.edu 